\title{
Multi-band printed PIFA Antenna with ground plane capacitive resonator
}

Benito Sanz-Izquierdo, John Batchelor and Richard Langley

This paper is a preprint of a paper accepted by Electronics Letters and is subject to Institution of Engineering and Technology Copyright. When the final version is published, the copy of record will be available at IET Digital Library 


\title{
Multi-band printed PIFA Antenna with ground plane \\ capacitive resonator
}

Benito Sanz-Izquierdo, John Batchelor and Richard Langley

\section{Indexing Terms:}

Antennas, multiband antennas, mobile communications

\begin{abstract}
$\underline{\text { Abstract: }}$
A novel multi-band PIFA antenna will be presented showing simulated modal current distributions and measured input impedance. All major mobile communications bands have been included and measurements show the radiation patterns to have good H-plane omni-directionality at all bands.
\end{abstract}

\section{Introduction}

The planar inverted-F antenna has been successfully used to design dual band antennas suitable for mobile communications systems, [1]. Additionally, it has been shown that parasitically coupled elements in plane with a top patch can be used to broadband antennas, [2]. This letter presents a multiband mobile system antenna with good quality radiation patterns at all bands. The antenna uses a novel technique to incorporate an extra band. The additional band is incorporated by using a parasitic element in the plane of the ground that is excited by coupling from the surrounding finite sized ground plane. When included in an existing dual band GSM/DCS antenna design, the resulting matched input bandwidth is sufficient to cover both the DECT and UMTS bands, an enlargement of more than 130\%. The effect of the ground plane parasitic element is demonstrated here by inclusion in a compressed size dual band antenna originally proposed by Viratelle and Langley, [3]. The antenna with a parasitic ground element remains compact, low-cost and is appropriate for the GSM850 (824 - 894 MHz), GSM900 (890-960 MHz), DCS1800 (1710-1880 MHz), DCS1900 (1850-1990 MHz) DECT (1880-1990 MHz) and UMTS (1900-2200 MHz) cellular telephone system applications. IE3D was used to simulate the antenna with good agreement found between the simulations and practical results. 


\subsection{Design guidelines}

The antenna is simple and low cost to construct with the metallic elements printed on a flexible material which is shaped around a honeycomb former. Figure 1 shows the geometry of the five-band printed PIFA Antenna with ground plane patch resonator, the honeycomb former has been omitted for clarity. As can be seen from Fig.1, the feeding probe is connected to the top inner patch element A and the two symmetrical top outer patches of element B connect through shorting walls to the ground plane C. The added parasite element $\mathrm{D}$ at the bottom plane is not electrically connected to the ground plane and has a rectangular shape with dimensions $15 \times 49.5 \mathrm{~mm}$. There is a $0.5 \mathrm{x} 10 \mathrm{~mm}$ feed line with a $0.5 \mathrm{~mm}$ air gap between the feed and the antenna ground element C. Elements A \& C provide the GSM900 band while B \& C control the DCS1800 band. Element D is the proposed new parasitic element that provides the DECT and UMTS bands in association with elements A \& C. An indication of the relative effects of the individual element lengths is given in Fig.2. Note that the parasitic patch element D allows the upper band to be tuned independently of the lower band which simplifies the tuning process. The inductance of the long narrow feed line between element $\mathrm{D}$ and ground element $\mathrm{C}$ is tuned at by a capacitive air gap and a broadband match is achieved for the upper frequencies of operation. The major antenna dimensions are given in table 1 and when the antenna is correctly designed, the fractional bandwidth of the GSM band is $18.8 \%$. The upper band spans from 1.69 $-2.21 \mathrm{MHz}$ which is sufficient to encompass the DCS, DECT and UMTS bands.

\subsection{Current Distributions}

The modal current distributions of the GSM and DCS bands for an antenna with no parasitic ground patch has been described in [3]. The fact that the rear ground plane is small means that a distinct modal distribution is observed in its outer legs (element $\mathrm{C}$ in Fig.1). The parasitic patch is placed between the ground plane legs to take advantage of the high current peaks which provide strong capacitive coupling. The current distribution shown in Fig.3a illustrates how the parasitic patch is not excited at the lower band, but in Fig. $3 \mathrm{~b}$ the patch becomes hot at the higher frequencies of the upper band. The loading effect of the patch on the outer legs of the ground plane 
serves to increase the upper bandwidth from $220 \mathrm{MHz}$ to $520 \mathrm{MHz}$ without affecting the lower band.

\subsection{Radiation patterns and gain measurements}

Measured H-plane patterns are given in Fig.4 for all the antenna bands, while an Eplane plot is shown in Fig.5. A PIFA antenna with an electrically long feed probe can be regarded as a top loaded monopole with a reduced size loading element. The radiation patterns are therefore expected to be those of a monopole in nature. Indeed, good omni-directionality is observed in all cases suggesting the antenna is well suited to its intended application as a picocell basestation antenna. The peak gain was also measured for all bands and was found to vary by only $0.5 \mathrm{dBi}$ across the GSM band. The gain variations across the DCS1800, DECT and UMTS bands are 3.3, 1.1 and 2 $\mathrm{dBi}$ respectively. The measured gain never fell significantly below $-1 \mathrm{dBi}$.

\subsection{Conclusion and further developments}

A dual-band PIFA antenna designed for GSM900 and DCS1800 has been enhanced to cover DECT and UMTS by the novel addition of a parasitic element in the ground plane. The parasitic element loads the small ground plane and increases the width of the upper band by $130 \%$ compared to the original antenna. The new antenna is vertically polarised presenting good omni-directional dipole patterns making it suitable for mobile picocell basestation applications. The gain remains stable over each band with a variation of $3.3 \mathrm{dBi}$ in the worst case and the measured gain was never observed to fall below $-1 \mathrm{dBi}$ in the operating bands.

\section{References:}

1 Dong Liu, Z.., Hall, P.S. and Wake, D.: "Dual-frequency planar inverted-F antenna”, IEEE Trans. Antennas Propag. 1997, 45, (10) pp. 1451-1457

2 C.K.Wu aand K.L. Wong "Broadband microstrip antenna with directly coupled and gap-coupled parasitic patches, "Microwave Opt. Technol. Lett 22 348349, Sep. 5. 1999 
3 Viratelle, D. and Langley, R.J.: "Dual-Band Printed Antenna for Mobile Telephone Applications”, IEE P-Micro., Anten. \& Propag., 147, (5), pp.381-384, Oct. 2000.

\section{Authors' affiliations:}

All authors are with the Electronics Department of the University of Kent, Canterbury, Kent, CT2 7NT.

Corresponding Author:

j.c.batchelor@kent.ac.uk 
Figure 1: Multiband PIFA antenna with parasitic ground element

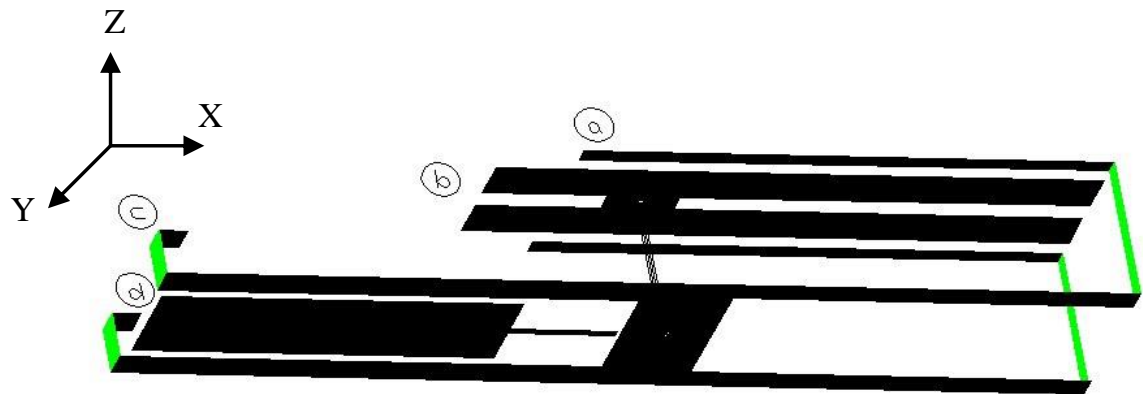


Figure 2: S11 plot indicating element parameter effect.

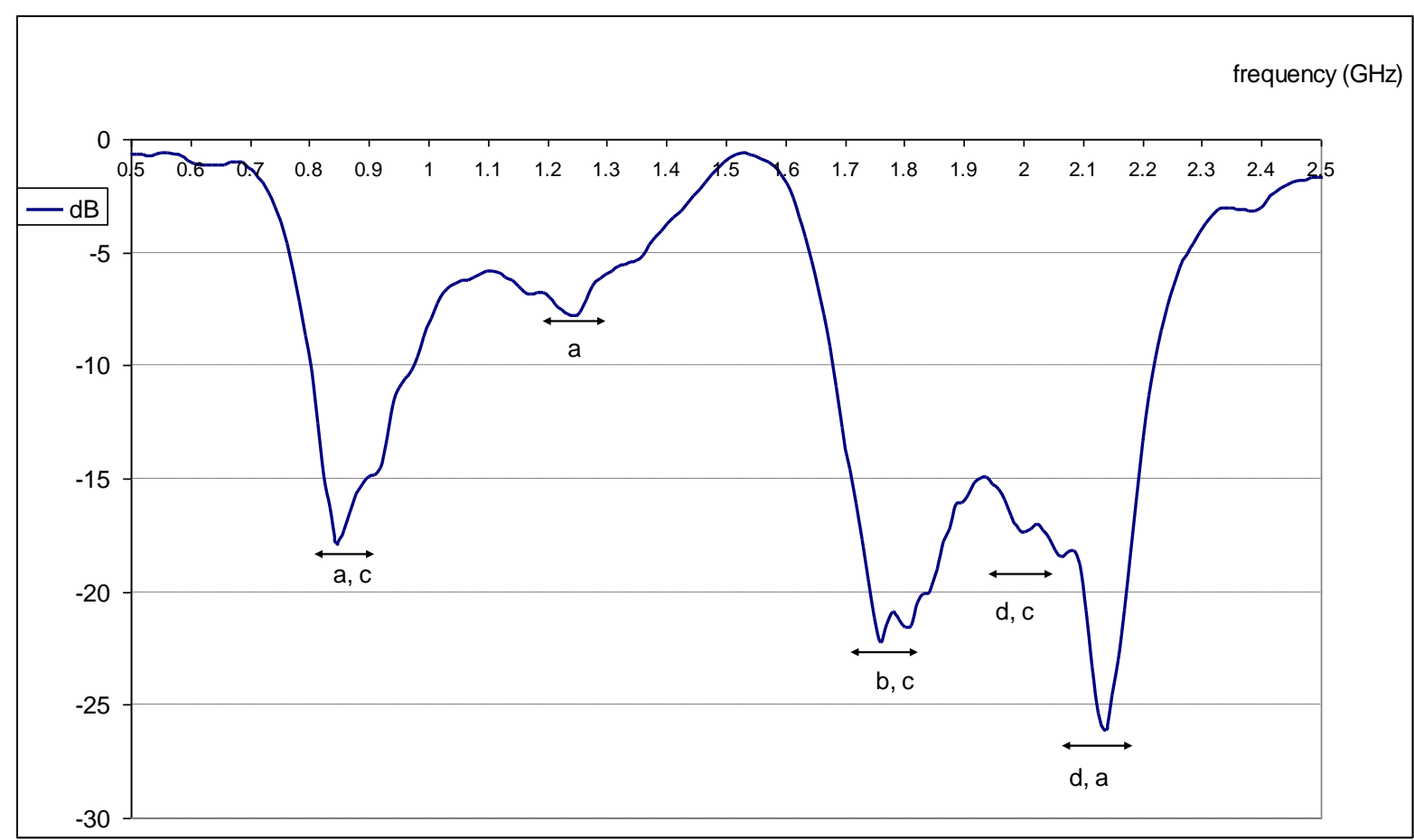


Figure 3: Multiband current distributions

(a) $900 \mathrm{MHz}$

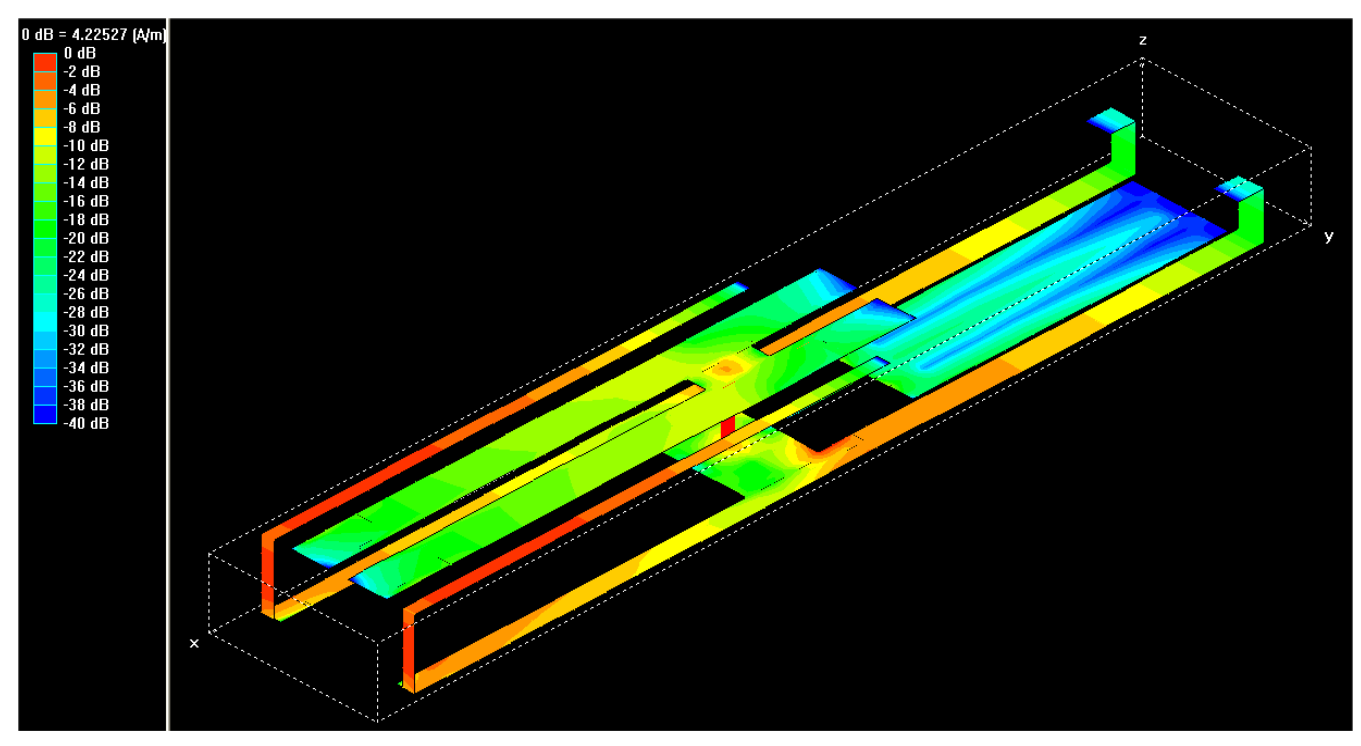

(b) $2.1 \mathrm{GHz}$

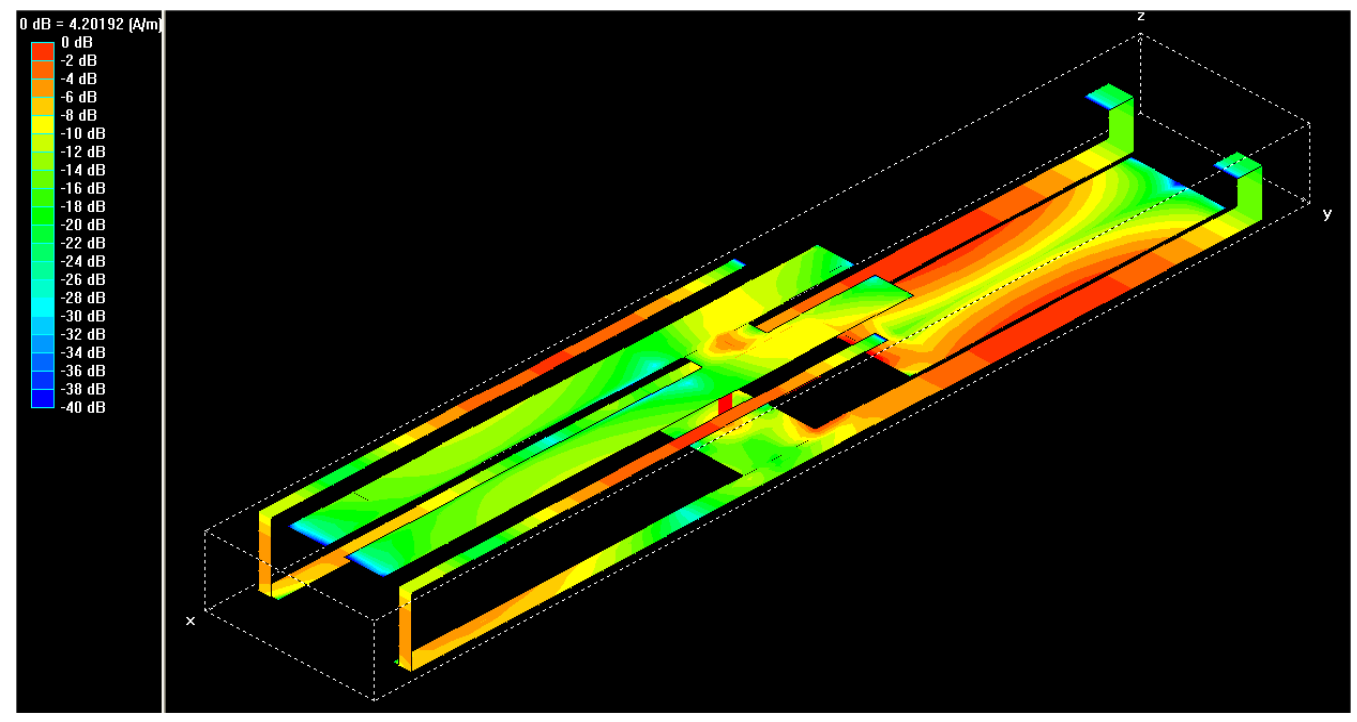


Figure 4: H-plane ( $y-z)$ radiation patterns for GSM900, DCS1800, DECT and UMTS bands.

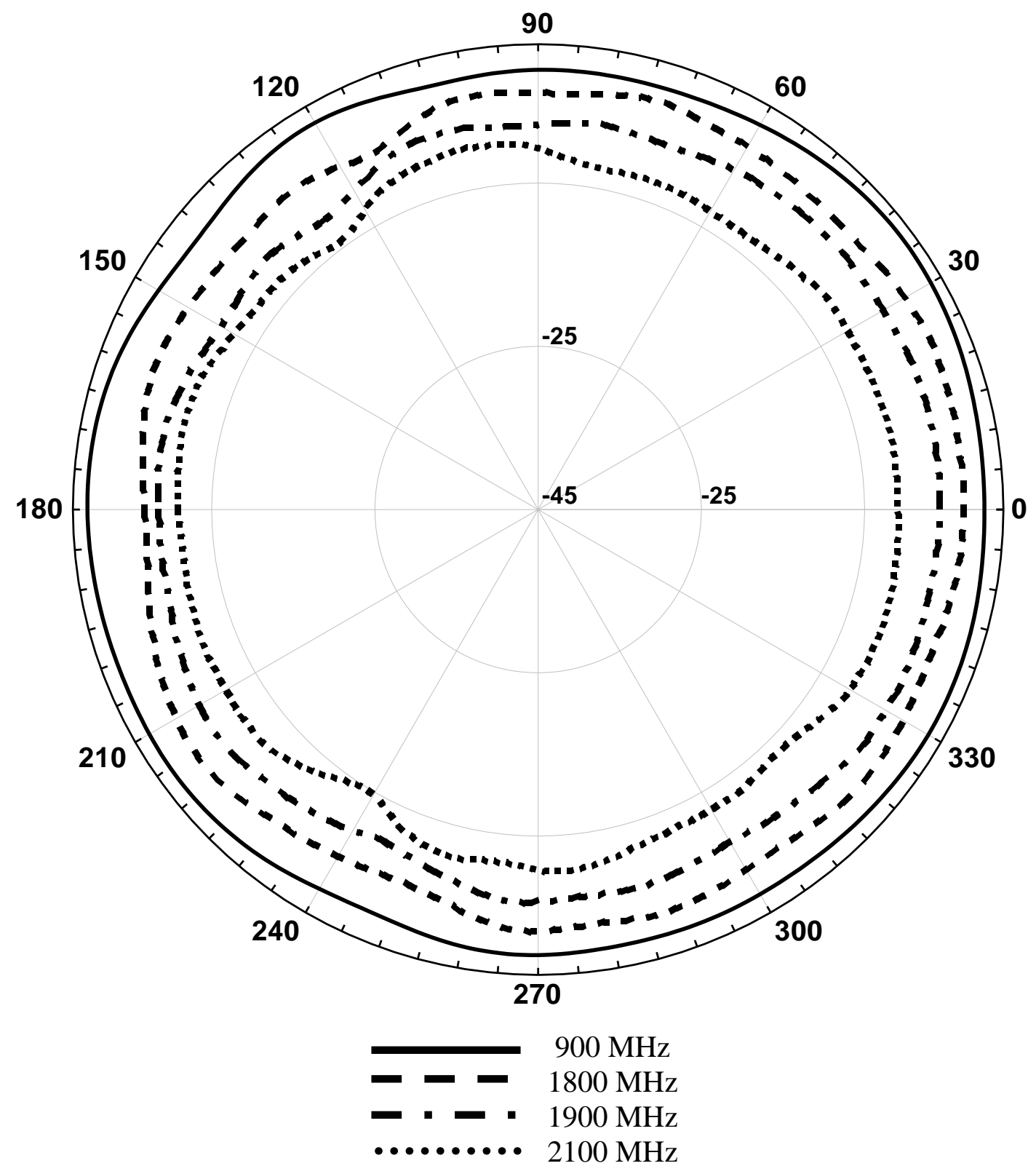


Figure 5: E-plane ( $x-z$ plane) radiation patterns for GSM900, DCS1800, DECT and UMTS bands.
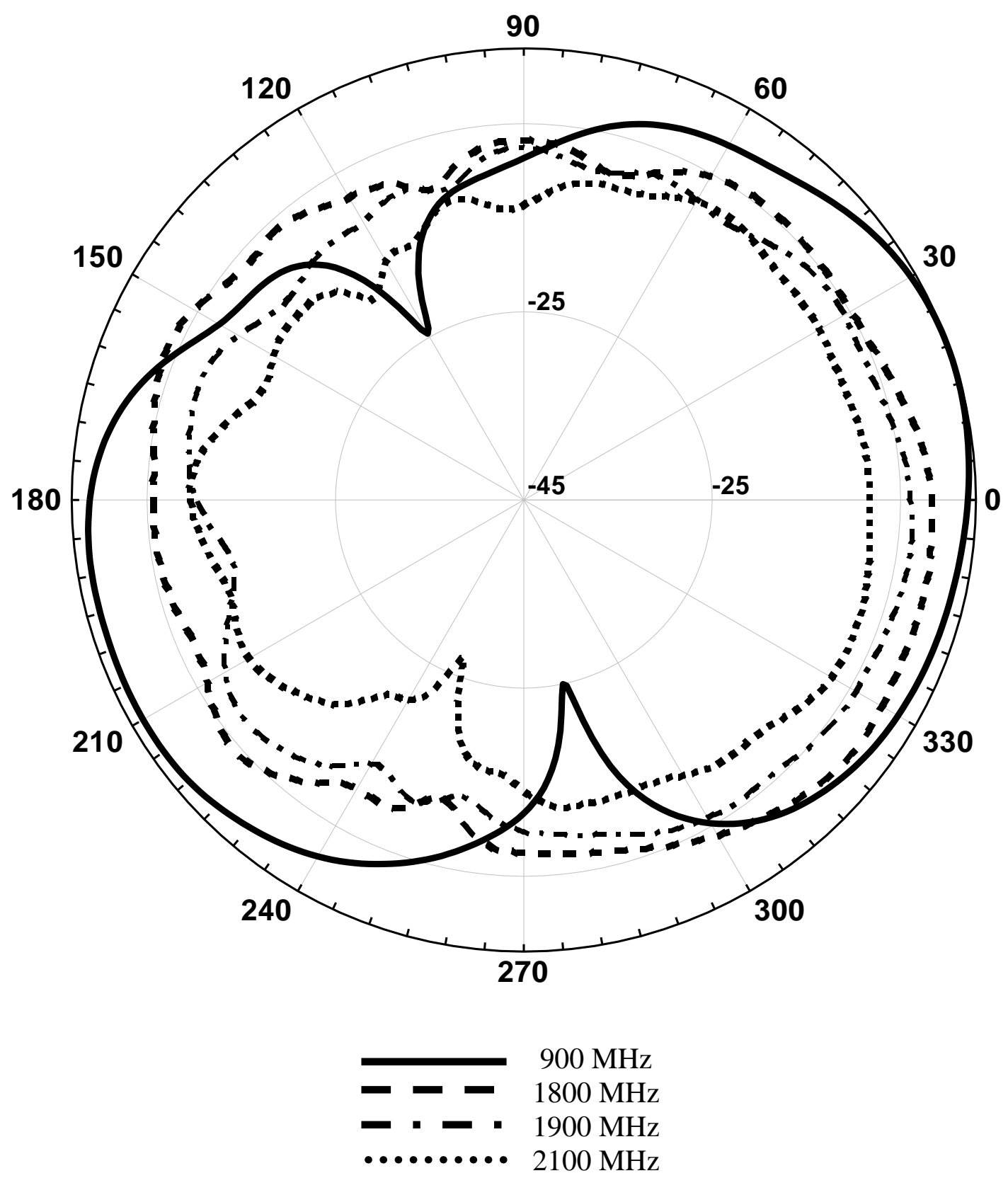
Table 1: Multiband antenna dimensions

\begin{tabular}{|c|c|c|c|c|}
\hline Element & A & B & C & D \\
\hline Width (mm) & 9.5 & 15 & 24 & 15 \\
\hline Length (mm) & 83 & 72.5 & 133 & 49.5 \\
\hline Height (mm) & \multicolumn{4}{|c|}{9.5} \\
\hline
\end{tabular}

